\title{
Diagnóstico bioquímico de la hiperprolactinemia monomérica
}

\section{Biochemical diagnosis of monomeric hyperprolactinemia}

\author{
A. Rivero, A. García-Calvo
}

\section{RESUMEN}

Fundamento. La prolactina se puede presentar bajo varias formas moleculares siendo la forma monomérica (PRLm) la biológicamente activa. La presencia de macroprolactina (MPRL) puede originar un falso diagnóstico de hiperprolactinemia debido a la interferencia en el procedimiento de medida. El objetivo ha sido desarrollar un protocolo que permita diagnosticar la hiperprolactinemia monomérica, que además sea complementario al procedimiento que detecta MPRL.

Material y métodos. La población de referencia para PRLm estaba formada por 122 mujeres y 140 hombres aparentemente sanos a los que se les extrajo sangre para la cuantificación de PRL. Además, se recogieron 49 sueros (33 mujeres y 16 hombres) hiperprolactinémicos. Se cuantificó PRL en todas las muestras en un Immulite 2000. La detección de MPRL y de PRLm se realizó tras precipitación con polietilenglicol. Se confirmó el resultado por cromatografía de filtración en gel. Para la obtención de los valores de referencia se siguieron las indicaciones del Panel de Expertos de la IFCC.

Resultados. Los valores de referencia de PRLm fueron 3,4$26,6 \mu \mathrm{g} / \mathrm{L}$ y $4,6-16,4 \mu \mathrm{g} / \mathrm{L}$ en mujeres y varones, respectivamente. De los 49 pacientes hiperprolactinémicos, en el 57 \% la concentración de PRLm tras PEG se encontraba fuera del intervalo de referencia previamente obtenido, confirmándose la presencia de hiperprolactinemia monomérica.

Conclusiones. Se ha desarrollado e implantado un protocolo para la cuantificación de PRLm. La obtención de los valores de referencia de PRLm permite el diagnóstico de la hiperprolactinemia monomérica o activa de forma complementaria a la identificación de MPRL.

Palabras clave. Prolactina. Prolactina monomérica. Macroprolactina. Valores de referencia. Polietilenglicol. \begin{abstract}
Background. Prolactin can take several molecular forms of which the most biologically active is the monomeric form (PRLm). The presence of macroprolactin (MPRL) can give rise to a false diagnosis of hyperprolactinemia due to interference in the measuring procedure. The aim was to develop a protocol that enables diagnosis of monomeric hyperprolactinemia, which should also be complementary to the procedure for detecting MPRL.
\end{abstract}

Material and methods. The reference population for PRLm was made up of 122 healthy women and 140 healthy men, from whom blood was extracted for PRL quantification. Additionally, 49 hyperprolactinemic serums (33 women and 16 men) were collected. PRL was quantified in all the samples in an Immulite 2000. The detection of MPRL and PRLm was carried out following precipitation with polyetylenglicol (PEG). The result was confirmed by gelatin filtration chromatography. The reference values were obtained following the indications of the Expert Panel of the IFCC.

Results. The PRLm reference values were 3,4-26,6 $\mu \mathrm{g} / \mathrm{L}$ and 4,6-16,4 $\mu \mathrm{g} / \mathrm{L}$ in women and men, respectively. In $57 \%$ of the 49 hyperprolactinemic patients the concentration of PRLm following PEG fell outside the previously obtained reference interval, confirming the presence of monomeric hyperprolactinemia.

Conclusions. A protocol for quantifying PRLm has been developed and implemented. Obtaining PRLm reference values makes it possible to diagnose monomeric or active hyperprolactinemia in a complementary form to the identification of MPRL.

Key words. Prolactin. Monomeric prolactin. Macroprolactin. Reference values. Polyethylene Glycol.

\section{An. Sist. Sanit. Navar. 2011; 34 (2): 145-152}

\section{Servicio de Bioquímica. Complejo Hospitalario} de Navarra

Recepción: 1 de marzo de 2011

Aceptación provisional: 6 de abril de 2011

Aceptación definitiva: 11 de abril de 2011

\section{Correspondencia}

Adriana Rivero Marcotegui

Servicio de Bioquímica

Complejo Hospitalario de Navarra

Irunlarrea, 3

31008 Pamplona

E-mail. ariverom@cfnavarra.es 


\section{INTRODUCCIÓN}

La prolactina (PRL) es una hormona peptídica que se puede presentar bajo tres formas moleculares ${ }^{1}$ : la forma monomérica (PRLm) biológicamente activa, la big prolactin de estructura no del todo conocida y la big-big prolactin o macroprolactina (MPRL) que es un complejo de PRL con inmunoglobulina $\mathrm{G}$ fundamentalmente, aunque también se ha comprobado la existencia de complejos con IgA o MPRL glicosilada ${ }^{2-4}$. En el sujeto sano, la forma monomérica representa el 70-90 \% de la PRL, mientras que las otras formas moleculares pueden alcanzar el $10 \%$ y el $5 \%$ para la big prolactin y para la big-big prolactin, respectivamente.

Una de las causas patológicas de elevación de PRL es el adenoma hipofisario o prolactinoma. El diagnóstico bioquímico pasa inexorablemente por la demostración de una concentración elevada de PRL en suero.

El concepto de MPRL fue introducido en 1981 por Whittaker en una paciente que, a pesar de tener hiperprolactinemia, carecía de síntomas como galactorrea, amenorrea o infertilidad ${ }^{5}$. La MPRL presenta baja biodisponibilidad y actividad biológica, sin embargo su presencia puede ocasionar una interferencia en los procedimientos de medida de la PRL, causando una aparente y falsa hiperprolactinemia. Existen pacientes en los que la macroprolactinemia por sí sola justifica la hiperprolactinemia detectada. Sin embargo, en otros casos pueden coexistir las dos situaciones, macroprolactinemia e hiperprolactinemia monomérica. Dado que la MPRL carece prácticamente de actividad biológica, en los últimos años se está recomendando enfocar el diagnostico de laboratorio no solo hacia la detección de la MPRL, sino hacia el reconocimiento y la detección de una elevada concentración de PRLm que es la molécula activa ${ }^{6,7}$. Para la detección de MPRL la precipitación con polietilenglicol (PEG) es el método de triaje por ser un método sencillo, efectivo y económico. El PEG se caracteriza por su capacidad para precipitar macromoléculas ${ }^{1,8-10}$. La cromatografía de filtración en gel (CFG) es el procedimiento de referencia y como tal, es largo, laborioso y caro $^{1,2}$ por lo que no se utiliza de forma rutinaria.

En este Servicio se informa desde el año 2004 de la presencia o ausencia de MPRL gracias al desarrollo y validación previa de un protocolo (Cálculo 1). Teniendo en cuenta que la forma molecular activa de la PRL es la forma monomérica, el objetivo de este trabajo ha sido el desarrollo de un procedimiento (Cálculo 2) que permita diagnosticar la hiperprolactinemia monomérica, para lo cual además se debe obtener previamente los valores de referencia de PRLm.

\section{MATERIAL Y MÉTODOS}

La población de referencia estaba formada por 122 mujeres con una media de edad de 48 años (18-89 años) y 140 varones, con una media de edad de 45 años (15-80 años). Los individuos de referencia cumplían los siguientes requisitos: concentración de PRL dentro del intervalo de referencia vigente hasta ese momento en nuestro Servicio (adoptado de Siemens Healthcare Diagnostics S.L.: mujeres: 4,6-37 $\mu \mathrm{g} / \mathrm{L}$; varones: $2,1-13 \mu \mathrm{g} / \mathrm{L}$ ), individuos eutiroideos, ausencia de analítica previa solicitada por los servicios de Ginecología y Obstetricia, Endocrinología y Psiquiatría, ausencia de medicación que modifique la concentración de PRL y ausencia de embarazo. Para la selección de estos individuos fue necesario el acceso a la historia clínica de los mismos, para lo cual se solicitó y se obtuvo el permiso correspondiente del Comité Ético de Investigación Clínica (CEIC) de Navarra.

Por otra parte, se seleccionaron 49 sueros hiperprolactinémicos pertenecientes a 49 pacientes, 33 mujeres [edad $\overline{\mathrm{x}}=36$ (1568 años)] y 16 varones [edad $\overline{\mathrm{x}}=48(15-81$ años)] a los que el clínico había solicitado la determinación de PRL. La concentración de PRL en esta población fue: $\overline{\mathrm{x}}=45,5 \mu \mathrm{g} / \mathrm{L}$, $\mathrm{s}=30,8$, rango $=14-162 \mu \mathrm{g} / \mathrm{L}$.

De cada paciente se extrajeron $9 \mathrm{~mL}$ de sangre fueron extraídos de la vena antecubital en tubo Vacutainer (ref. 455010, Becton, Dickinson, NJ, EEUU). La sangre se centrifugó a $1.700 \mathrm{~g}$ durante 5 minutos y el 
suero fue analizado inmediatamente en el caso de la población hiperprolactinémica, o fue alicuotado y congelado a $-20^{\circ} \mathrm{C}$ hasta el momento de su determinación en el caso de la población de referencia.

\section{Procedimientos}

La cuantificación de PRL se realizó por quimioluminiscencia en un Immulite 2000 XPi (ref. L2KPR2, Siemens Healthcare Diagnostics S.L., Deesfield, EEUU).

Todos los sueros fueron tratados con polietilenglicol conforme a la literatura ${ }^{8,10,11}$ con el fin de obtener los valores de referencia de PRL monomérica (en el caso de la población de referencia), o de comprobar la existencia de MPRL o hiperprolactinemia monomérica (en el caso de la población hiperprolactinémica).

Cuantificación de MPRL (Cálculo 1): la concentración de PRL se expresa como porcentaje de recuperación de una dilución del suero con PEG, comparándola con el resultado obtenido en otra dilución similar del suero en agua destilada: ([PRLSobrenadante PEG]x 100)/[PRLSobrenadante Agua] = \% Recuperación.

Si el porcentaje de recuperación es menor del 54 \% la macroprolactina está presente en el suero, si el porcentaje de recuperación es mayor del 75 \% la macroprolactina está ausente, y si el porcentaje de recuperación oscila entre 54 y $75 \%$ el resultado no es concluyente y se debe realizar la $\mathrm{CFG}^{11}$.

Cuantificación de PRLm (Cálculo 2): se obtiene la concentración de PRL monomérica: [PRLSobrenadante PEG] x $2=[$ PRLm $(\mu \mathrm{g} / \mathrm{L})]$.

Si la concentración de PRL monomérica se encuentra dentro del intervalo de refe- rencia de PRLm previamente obtenido se descarta la existencia de hiperprolactinemia monomérica.

La cromatografía de filtración en gel (CFG) fue empleada como técnica de referencia para la detección de MPRL ${ }^{1,11}$. El gel fue Sephadex G-200 (ref. 1700801, Amersham Pharmacia Biotech, Buckinghamshire, Inglaterra) empaquetado en una columna de $1 \times 20 \mathrm{~cm}$ conectada a un colector de fracciones (LKB Wallac, Turku, Finlandia). Así, $500 \mu \mathrm{L}$ de suero fueron introducidos en la columna previamente equilibrada con tampón Tris (50 mM, pH 7,2) (ref. 1319410, Panreac, Barcelona, España). El flujo fue de $0,125 \mathrm{~mL} / \mathrm{min}$ y se recogieron fracciones de $120 \mu \mathrm{L}$ por suero aplicado. La concentración de PRL fue medida en cada fracción.

Análisis estadístico. Se aplicó estadística descriptiva y la comparación entre grupos se realizó mediante la prueba U de Mann Whitney. Los valores de referencia se obtuvieron siguiendo las indicaciones del Panel de Expertos de la IFCC ${ }^{12}$.

\section{RESULTADOS}

\section{Valores de referencia de PRL}

Se midió la concentración de PRL en los 262 individuos integrantes de la población de referencia. Se observó que existían diferencias significativas entre sexos $(\mathrm{U}=6457 ; \mathrm{p}=0,001)$ por lo que se partió la población atendiendo a este criterio. Se comprobó si en la población existían datos aberrantes mediante la aplicación del método de los múltiplos de la desviación. En las mujeres se encontraron 2 datos aberrantes que fueron eliminados. Tras comprobar la distribución de datos de la población se obtuvieron los valores de referencia (Tabla 1).

Tabla 1. Valores de referencia de PRL

\begin{tabular}{c|ccc}
\hline PRL $(\boldsymbol{\mu g} / \mathbf{L})$ & $\mathbf{F}_{\mathbf{0 , 0 2 5}} \mathbf{I C ~ 9 0 ~ \% )}$ & $\mathbf{F}_{\mathbf{5 0}}$ & $\mathbf{F}_{\mathbf{0 , 9 7 5}} \mathbf{( I C ~ 9 0 ~ \% )}$ \\
\hline Mujeres & $2,59(1,4-3,2)$ & 7,82 & $22,61(17,7-24,4)$ \\
\hline Varones & $2,85(2,3-3,3)$ & 6,18 & $13,82(12,1-16,2)$ \\
\hline
\end{tabular}

PRL: prolactina; $\mathrm{F}_{0,025}$ : fractil 0,$025 ; \mathrm{F}_{50}$ : fractil $50 ; \mathrm{F}_{0,975}$ : fractil 0,975 ; IC: intervalo de confianza. 


\section{Valores de referencia de PRL monomérica tras PEG}

Se realizó el cálculo 2 obteniendo la concentración de PRLm en los sueros de los 262 individuos de la población de referencia. Una vez comprobado que existían diferencias significativas entre sexos $(U=6417$; $\mathrm{p}=0,001)$ se partió la población atendiendo a este criterio. Dos datos aberrantes correspondientes a mujeres fueron eliminados. Los valores de referencia de PRLm en nuestra población se muestran en la tabla 2 y se obtuvieron tras la aplicación de estadística no paramétrica, ya que la población no seguía una distribución normal.

Tabla 2. Valores de referencia de PRLm

\begin{tabular}{c|ccc}
\hline PRL $(\boldsymbol{\mu g} / \mathbf{L})$ & $\mathbf{F}_{\mathbf{0 , 0 2 5}}(\mathbf{I C ~ 9 0} \%)$ & $\mathbf{F}_{\mathbf{5 0}}$ & $\mathbf{F}_{\mathbf{0}, \mathbf{9 7 5}}$ (IC 90 \%) \\
\hline Mujeres & $3,35(2,1-4,1)$ & 10,41 & $26,56(21,4-29,2)$ \\
\hline Varones & $4,46(3,6-4,8)$ & 8,23 & $16,44(14,6-19,7)$ \\
\hline
\end{tabular}

PRL: prolactina; $\mathrm{F}_{0,025}$ : fractil 0,$025 ; \mathrm{F}_{50}$ : fractil $50 ; \mathrm{F}_{0,975}:$ fractil 0,$975 ;$ IC: intervalo de confianza.

En ambos sexos, el límite superior del intervalo de referencia es mayor en la PRLm tras precipitación con PEG que en la PRL sérica total. La recuperación media de PRL en esta población de referencia tras precipitación con PEG fue $115 \%$ (42-175\%). Se observa por tanto una interferencia positiva (recuperación $>100 \%$ ) del PEG en el procedimiento de cuantificación de PRL en el analizador Immulite 2000.

\section{Prolactina en la población hiperprolactinémica}

Los sueros de los 49 individuos hiperprolactinémicos fueron tratados con PEG a fin de poder detectar la presencia de MPRL y/o hiperprolactinemia monomérica. La recuperación de PRL tras precipitación con PEG superó el 100\% en algunos casos $[\bar{x}=90 \%(8-159 \%)]$. Se encontraron diferencias significativas $(\mathrm{U}=4632, \mathrm{p}=$ $0,002)$ en la recuperación tras PEG entre la población normo e hiperprolactinémica.

De los 49 pacientes hiperprolactinémicos, $15(31 \%)$ presentaron un porcentaje de recuperación menor del 54\%. Según el cálculo 1, ya implantado en nuestro servicio, estos pacientes serían clasificados como macroprolactinémicos. En 33 pacientes (67\%) la presencia de MPRL sería descartada, mientras que en el paciente restante (2\%) se debería realizar CFG al haber obtenido un resultado no concluyente ${ }^{11}$.

Conforme al nuevo cálculo 2 , en 28 pacientes (57\%) la concentración de PRLm tras PEG se encontraba fuera del intervalo de referencia previamente obtenido (Tabla 2), confirmándose la presencia de hiperprolactinemia monomérica.

En el 84\% de los 49 pacientes hiperprolactinémicos, cuando había macroprolactinemia no había hiperprolactinemia monomérica y viceversa. Sin embargo, hubo 8 casos reseñables por no seguir este patrón (Tabla 3). El caso 7 presentó MPRL e hiperprolactinemia monomérica. En el caso 19, el cálculo 1 produjo un resultado no concluyente y el cálculo 2 descartó la hiperprolactinemia monomérica. Sorprendentemente, los casos hiperprolactinémicos $20,25,26,27,29$ y 43 no tenían ni MPRL ni hiperprolactinemia monomérica según los cálculos 1 y 2 , respectivamente. Se pudo realizar CFG como técnica de referencia en 6 de los 8 sueros. Los resultados más representativos se muestran en la figura 1. Tras la CFG se observó la presencia de macroprolactina en los casos 7, 19 y 29 (Fig. 1). 
Tabla 3. Casos caracterizados por no seguir el patrón de comportamiento mayoritario

\begin{tabular}{|c|c|c|c|c|c|c|c|c|}
\hline Caso & Sexo & $\begin{array}{l}\text { PRL } \\
(\mu \mathrm{g} / \mathrm{L})\end{array}$ & $\begin{array}{c}\% \mathrm{R} \\
\mathrm{Cl}\end{array}$ & $\begin{array}{c}\text { Presencia } \\
\text { de MPRL } \\
\text { Cl }\end{array}$ & $\begin{array}{c}\text { PRLm } \\
(\mu \mathrm{g} / \mathrm{L}) \\
\mathrm{C} 2\end{array}$ & $\begin{array}{c}\text { Hiperprolactinemia } \\
\text { monomérica } \\
\text { C2 }\end{array}$ & $\begin{array}{l}\text { CFG de } \\
\text { Presencia } \\
\text { MPRL }\end{array}$ & $\begin{array}{l}\text { Componente } \\
\text { mayoritario en } \\
\text { CFG }\end{array}$ \\
\hline 7 & $\mathrm{M}$ & 60,7 & 40 & SI & 27,8 & SI & SI & MPRL \\
\hline 19 & $\mathrm{M}$ & 30,4 & 60 & ¿? & 22,2 & NO & SI & PRLm \\
\hline 20 & $\mathrm{M}$ & 30,2 & 117 & NO & 24,2 & NO & - & - \\
\hline 25 & $\mathrm{M}$ & 25,4 & 97 & NO & 25,6 & NO & - & - \\
\hline 26 & $\mathrm{M}$ & 25,2 & 110 & NO & 21,2 & NO & NO & PRLm \\
\hline 27 & $\mathrm{M}$ & 25,1 & 116 & NO & 21,4 & NO & NO & PRLm \\
\hline 29 & $\mathrm{M}$ & 24,2 & 99 & NO & 18,9 & NO & SI & PRLm \\
\hline 43 & V & 14,2 & 126 & NO & 14,4 & NO & NO & PRLm \\
\hline
\end{tabular}

PRL: prolactina; \% R: porcentaje de recuperación; C1: cálculo 1; C2: cálculo 2; CFG: cromatografía de filtración en gel.
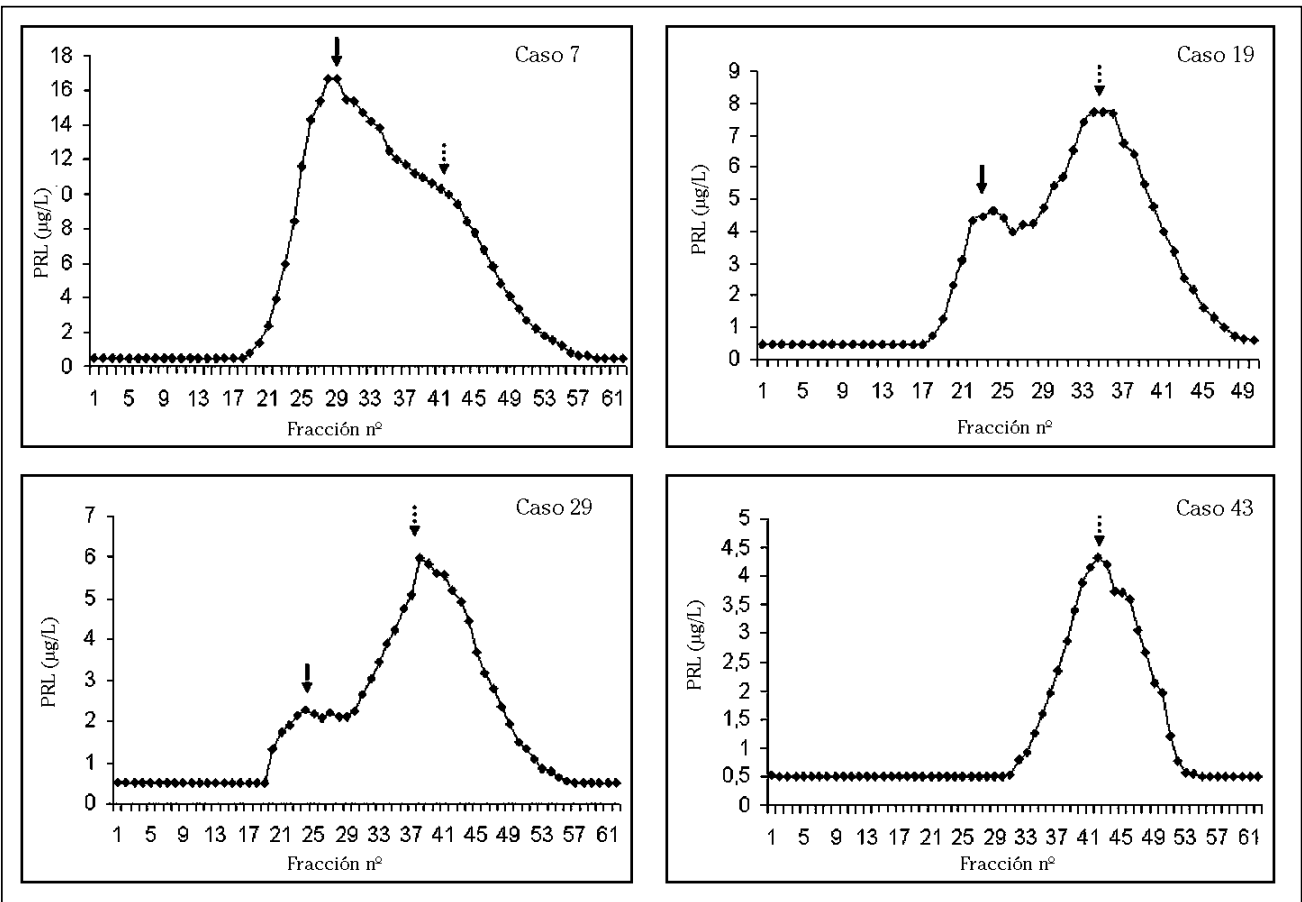

Flecha continua: MPRL. Flecha discontinua: PRLm.

Figura 1. CFG de 4 sueros hiperprolactinémicos.

\section{DISCUSIÓN}

La macroprolactina es un complejo de PRL que es aclarado de la circulación lentamente ocasionando una aparente hiper- prolactinemia $^{8}$. Se cree que su tamaño hace imposible que atraviese las membranas capilares, de forma que in vivo tiene una actividad biológica muy limitada ${ }^{13,14}$. Aunque algunos autores ${ }^{9,15}$ han relacionado la ma- 
croprolactinemia con la presencia de adenomas y síntomas de hiperprolactinemia, para otros estos hallazgos están ausentes ${ }^{16,17}$. En un trabajo recientemente publicado se ha comprobado que pacientes diagnosticadas de macroprolactinemia y sometidas a un largo seguimiento de casi 10 años, no sufrieron progresión sintomática, y por tanto a juicio de estos autores se hacen innecesarias extensas revisiones de estos pacientes ${ }^{18}$.

Aunque la MPRL carezca prácticamente de actividad biológica in vivo, su presencia puede originar in vitro resultados falsamente elevados de PRL, de ahí la importancia de su detección. No obstante, la presencia de MPRL no descarta la presencia de hiperprolactinemia monomérica ya que ambas formas moleculares pueden coexistir en un mismo paciente. Desde hace años se recomienda la detección de MPRL en los sueros hipeprolactinémicos ${ }^{18,19} \mathrm{y}$ en pacientes con prolactinoma ${ }^{20}$. Igualmente se recomienda la obtención de los valores de referencia de PRL monomérica como procedimiento que permita, no ya detectar la presencia de MPRL sino diagnosticar una verdadera hiperprolactinemia monomérica, con independencia de la presencia o no de MPRL, de forma que se informe por tanto de la concentración de la fracción activa o monomérica $^{6,7}$. Con el procedimiento implantado en nuestro servicio (cálculo 1) únicamente se informaba de la presencia o ausencia de MPRL, sin tener en cuenta que la presencia de MPRL no es incompatible con la presencia de PRLm en alta concentración ${ }^{6,21}$. Por ello, y atendiendo a las recomendaciones actuales se ha implantado además el cálculo 2 que informa de la presencia o ausencia de hiperprolactinemia monomérica ${ }^{7}$.

Según la bibliografía, la prevalencia de la macroprolactinemia oscila entre 11-42\%, dependiendo entre otros causas del procedimiento utilizado ${ }^{10,11}$, ya que la MPRL presenta una reactividad variable en los inmunoanálisis ${ }^{22-24}$. Así, los procedimientos son clasificados como de baja, media y elevada reactividad con la macroprolactina ${ }^{24,25}$. El inmunoanálisis por quimioluminiscencia en un Immulite 2000 es considerado como un método de detección media ${ }^{24}$.
El procedimiento de precipitación con PEG presenta algunos inconvenientes. El PEG interfiere con los inmunoanálisis en mayor o menor grado ${ }^{24}$. Esta interferencia puede ser debida a la concentración de PEG, a su fuerza iónica y/o a la temperatu$\mathrm{ra}^{8}$. A este respecto, se han propuesto alternativas para evitar dicha interferencia, como es la de tratar los calibradores de la técnica con PEG aunque con resultados contradictorios $^{26,27}$. El PEG precipita además cierta cantidad de PRLm sobreestimando la concentración de MPRL ${ }^{6,28}$.

En este trabajo se han obtenido, aunque no era el objetivo del mismo, los valores de referencia propios de PRL (mujeres $2,6-22,6 \mu \mathrm{g} / \mathrm{L}$; varones $2,8-13,8 \mu \mathrm{g} / \mathrm{L}$ ) que han sido incorporados en nuestro servicio. La diferencia entre los valores de referencia propios y los que presenta el fabricante forman parte de la práctica diaria, y simplemente puede estar justificada por las diferentes poblaciones estudiadas (número de individuos, sexo, criterios de selección, etc.). Los valores de referencia de los fabricantes varían ostensiblemente, así por ejemplo, en la encuesta de United Kingdom National External Quality Assessment Scheme hubo diferencias de hasta el $30 \%$ en los resultados de PRL obtenidos con diferentes analizadores, a pesar de la utilización por todos ellos del mismo estándar internacional IS $84 / 500^{29}$.

Para diagnosticar a un paciente de hiperprolactinemia monomérica es requisito imprescindible obtener previamente los valores de referencia de PRL monomérica tras PEG que en nuestro caso fueron 3,4$26,6 \mu \mathrm{g} / \mathrm{L}$ y $4,5-16,4 \mu \mathrm{g} / \mathrm{L}$ en mujeres y varones, respectivamente. Estos resultados difieren ligeramente con los publicados en la literatura, lo cual puede obedecer igualmente a los diferentes inmunoensayos y poblaciones utilizadas ${ }^{6,7,30}$. El hecho de que los valores de referencia de PRLm sean más altos que los de PRL total podría justificarse por la interferencia del PEG en el immunoanálisis.

De los 49 pacientes hiperprolactinémicos, 41 tenían o macroprolactinemia o hiperprolactinemia monomérica. Los 
8 restantes son recogidos en la tabla 3 y mostraron una clasificación diferente. Clínicamente lo mas reseñable en ellos era: hipotiroidismo leve (caso 19), hirsutismo idiopático (caso 20), ovario poliquístico (caso 25), hiperandrogenismo de origen ovárico (caso 26), macroprolactinoma posiblemente no funcionante (caso 27) y disfunción eréctil (caso 43). El caso 7 según el cálculo 1 tenía MPRL y según el cálculo 2 tenía también hiperprolactinemia monomérica, lo cual fue además confirmado tras CFG. Se evidencia por tanto, que pueden existir pacientes cuya elevada concentración de PRL esté justificada tanto, por un aumento de PRL monomérica como por la presencia de MPRL ${ }^{8,30}$, por lo que los cálculos 1 y 2 pueden ser complementarios y no excluyentes. El resultado del cálculo 1 en el caso 19 no fue concluyente al obtener un porcentaje de recuperación entre $54 \mathrm{y}$ $75 \%$, y el cálculo 2 descartó la hiperprolactinemia monomérica. Tras CFG se comprobó que tenía ambos componentes con predominio de PRLm. Finalmente, los otros seis pacientes eran según los cálculos 1 y 2 hiperprolactinémicos sin MPRL y sin hiperprolactinemia monomérica. Este error en la clasificación podría obedecer a las siguientes causas: a) estos pacientes presentaban muy ligeras elevaciones de la concentración de PRL, b) la técnica de precipitación con PEG presenta una imprecisión intraserie entre 2,7 y $5,1 \%$ que puede justificar que a estas concentraciones borderline los resultados no sean muy concordantes ${ }^{11}$, c) hay también que tener en cuenta que el PEG y la CFG pueden producir resultados discordantes entre sí ya que cierta cantidad del monómero precipita con el PEG y d) se han detectado resultados falsos negativos en caso de que la PRL se una a IgA para formar el macrocomplejo ${ }^{4}$.

Según Suliman y col$^{6}$ la hiperprolactinemia atribuida a la MPRL es causa frecuente de diagnóstico y tratamiento erróneo de pacientes. Este problema podría ser evitado si los laboratorios aplicaran una prueba de escrutinio a todas las muestras con hiperprolactinemia para detectar la presencia de MPRL e informar de la concentración de PRLm o bioactiva. La coexistencia de macroprolactinemia e hiperprolactinemia monomérica es un hecho poco habitual, sin embargo enfocar únicamente la labor diagnóstica hacia la búsqueda de MPRL es un enfoque incompleto. Este nuevo procedimiento permite conocer, independientemente de la presencia de macroprolactina si existe hiperprolactinemia monomérica, y por tanto contribuye a un diagnóstico más claro que evitará pruebas de imagen y tratamientos innecesarios.

\section{BIBLIOGRAFÍA}

1. Comisión de interferencias y efectos de los medicamentos. Recomendaciones sobre las interferencias por macroprolactina en la medición de prolactina. Sociedad Española de Bioquímica Clínica. Comité Científico. Química Clínica 2006; 25: 45-49.

2. Fahie Wilson MN, John R, Ellis AR. Macroprolactin; high molecular mass forms of circulating prolactin. Ann Clin Biochem 2005; 42: 75-92.

3. Diver MJ, Ewins DL, Worth R, Bowles S, Ahlquist J, FAHIE-WILSON MN et al. An unusual form of big, big (macro) prolactin in a pregnant patient. Clin Chem 2001; 47: 346-348.

4. Kilvington F, Rahim A, Kavanagh L, Smith T, Fahie WILSON M. False negative PEG precipitation test in a case of hyperprolactinaemia with an IgA macroprolactin. Ann Clin Biochem 2006; 43 (Suppl 1):A35.

5. WhitTAKer PG, Wilson T, Lind T. Maintained fertility in a patient with hyperprolactinemia due to big big prolactin. J Clin Endocrinol Metab 1981; 53: 833-836.

6. Suliman AM, Smith TP, Gibney J, Mckenna TJ. Frequent misdiagnosis and mismanagement of hyperprolactinemic patients before the introduction of macroprolactin screening: application of a new strict laboratory definition of macroprolactinemia. Clin Chem 2003; 49: 1504-1509.

7. Beltran L, Fahie-Wilson MN, Mckenna TJ, KavaNAGH L, SMiTh TP. Serum total prolactin and monomeric prolactin reference intervals determined by precipitation with polyethylene glycol: evaluation and validation on common immunoassay platforms. Clin Chem 2008; 54 : 1673-1681.

8. FAHIE-WILSON MN. Macroprolactinaemia: contribution to hyperprolactinaemia in a district general hospital and evaluation of a 
screening test based on precipitation with polyethylene glycol. Ann Clin Biochem 1997; 34: 252-258.

9. Olukoga A, Kane J. Macroprolactinaemia: validation and application of the polyethylene glycol precipitation test and clinical characterization of the condition. Clin Endocrinol 1999; 51: 119-126.

10. VIEIRA JG. Extensive experience and validation of polyethylene glycol precipitation as a screening method for macroprolactinemia. Clin Chem 1998; 44: 1758-1759.

11. Rivero A, Alonso E, Grijalba A. Decision cut-off of the polyethylene glycol precipitation technique in screening for macroprolactinemia on Immulite 2000. Clin Chem Lab Med 2004; 42: 566-568.

12. Solberg HE. Establishment and Use of Reference Values. En: Burtis CA, Ashwood ER, editor. Tietz Textbook of Clinical Chemistry. Philadelphia: Saunders, 1994: 454-484.

13. Leaños-Miranda A, Chávez-Rueda KA, BlancoFAVELA F. Biologic activity and clearance of prolactin-IgG complex in patients with systemic lupus erythematosus. Arthritis Rheum 2001; 44: 866-875.

14. HatTori N. Anti-Prolactin (PRL) Autoantibodies cause asymptomatic hyperprolactinemia: bioassay and clearance studies of PRLImmunoglobulin G complex. J Clin Endocrinol Metabol 1997; 82: 3107-3110.

15. Vallette-Kasic S, Morange I, Selim A, Gunz G, Morange S, Enjalbert A, et al. Macroprolactinemia revised: A Study on 106 Patients. J Clin Endocrinol Metab 2001; 87: 581-588.

16. Lesle H, Courtney C, Bell P, Hadden D, Mccance D, EluIS P, et al. Laboratory and clinical experience in 55 patients with macroprolactinemia identified by a simple polyethylene glycol precipitation method. J Clin Endocrinol Metab 2001; 86: 2743-2746.

17. Fideleff HL, Ruibal G, Boquete H, Pujol A, SequeRA A, Sobrado P. Macroprolactinemia in childhood and adolescence: a cause of asymptomatic hyperprolactinemia. Horm Res 2000; 53:16-19.

18. Wallace R, Satti N, Courtney CH, Leslie $\mathrm{H}$, Bell PM, Hunter SJ, et al. Ten-year clinical follow-up of a cohort of 51 patients with macroprolactinemia establishes it as a benign variant. J Clin Endocrinol Metab 2010; 95: 3268-3271.
19. McKenNa TJ. Should macroprolactin be measured in all hyperprolactinaemic sera. Clin Endocrinol 2009; 71: 466-469.

20. Casanueva FF, Molitch ME, Schlechte JA, Abs R, Bonert V, Bronstein MD et al. Guidelines of the Pituitary Society for the diagnosis and management of prolactinomas. Clin Endocrinol 2006; 65: 265-273.

21. FAhIE-Wilson MN, Ahlquist JA. Hyperprolactinaemia due to macroprolactins: some progress but still a problem. Clin Endocrinol 2003; 58: 683-685.

22. John R, MC Dowell IF, Scanlon MF, Eldis AR. Macroprolactin reactivities in prolactin assays: an issue for clinical laboratories and equipment manufacturers Clin Chem 2000; 46: 884885.

23. FAHIE-WILSON MN. Detection of macroprolactin causing hyperprolactinemia in commercial assays for prolactin. Clin Chem 2000; 46: 2022-2023.

24. Gilson G, Schmit P, Thix J, Hoffman JP, Humbel RL. Prolactin results for samples containing macroprolactin are method and samples dependent. Clin Chem 2001; 47: 331-333.

25. Ellis AR, Fahie-Wilson MN, Axcell M, SANDs K, HILL RP. Macroprolactin(s): composition and reactivity in immunoassays and laboratory interpretation of results of an unusual patient serum. Ann Clin Biochem 2006; 43: 57-62.

26. Li W, Sustarsic D, Fahie Wilson M, Vankrieken L, Quero K, Del Rosario I et al. Matrix effect of PEG precipitation in detection of macroprolactin in Immulite and Immulite 2000 Prolactin assay. Clin Chem 2002; 48 (Suppl 6):A62.

27. Rivero A. Polyethylene glycol interference in the Immulite prolactin assay: treatment of the calibrators with PEG does not improve diagnostic accuracy. Clin Chem Lab Med 2008; 46: 296-297.

28. Kavanagh L, Mckenna TJ, Fahie-Wilson MN, GiBNEY J, SMITH TP. Specificity and clinical utility of methods for the detection of macroprolactin. Clin Chem 2006; 52: 1366-1372.

29. Ellis A, FAHIE Wilson M. Hyperprolactinaemia caused by antipsychotic drugs: a new application for prolactin assay. Ann Clin Biochem 2010; 47: 287-289.

30. Jassam NF, Paterson A, Lippiatt C, Barth JH. Macroprolactin on the Advia Centaur: experience with 409 patients over a three-year period. Ann Clin Biochem 2009; 46: 501-504. 